CARNETS DE Carnets de géographes

GÉOGRAPHES.

$7 \mid 2014$

Les espaces de l'entre-deux

\title{
La fabrication plurielle de centralités dans la périphérie de Constantine
}

Le cas de la ville nouvelle Ali Mendjeli

Ahcene Lakehal

(2) OpenEdition

Journals

Édition électronique

URL : http://journals.openedition.org/cdg/465

DOI : $10.4000 /$ cdg.465

ISSN : 2107-7266

Éditeur

UMR 245 - CESSMA

Référence électronique

Ahcene Lakehal, «La fabrication plurielle de centralités dans la périphérie de Constantine », Carnets de géographes [En ligne], 7| 2014, mis en ligne le 01 décembre 2014, consulté le 24 septembre 2020. URL : http://journals.openedition.org/cdg/465 ; DOI : https://doi.org/10.4000/cdg.465

\section{(c) (i) (2) $\Theta$}

La revue Carnets de géographes est mise à disposition selon les termes de la Licence Creative Commons Attribution - Pas d'Utilisation Commerciale - Pas de Modification 4.0 International. 


\title{
LA FABRICATION PLURIELLE DE CENTRALITÉS DANS LA PÉRIPHÉRIE DE CONSTANTINE Le cas de la ville nouvelle Ali Mendjeli
}

\begin{abstract}
AHCENELAKEHAL
La ville de Constantine, la troisième agglomération par la taille d'Algérie, a connu depuis le début des années 1980 une croissance démographique extrêmement rapide et un étalement considérable de ses périphéries urbanisées. Ce phénomène de l'étalement qui l'affecte, au même titre que toutes ses homologues les grandes villes et métropoles maghrébines, s'est accompagné de changements profonds dans son organisation socio-spatiale, dans les manifestations de son urbanité et dans les représentations que ses habitants se forgent des différents quartiers qui la composent. Il a abouti à la création de la Ville Nouvelle Ali Mendjeli qui constitue aujourd'hui, sans nul doute, l'un des principaux théâtres des dynamiques territoriales de la périphérie constantinoise.
\end{abstract}

Créée ex nihilo, située à $20 \mathrm{~km}$ à vol d'oiseau au sud de Constantine, la Ville Nouvelle Ali Mendjeli a été difficilement mise en place en 1999par les seules autorités locales (le wali et les services de la wilaya). Elle lui a fallu presque dix ans pour que son développement atteigne son rythme de croisière, grâce, il faut le reconnaître, à d'importants investissements de l'Etat, lesquels, pour être tardifs n'en ont pas moins été massifs. Aujourd'hui, la Ville Nouvelle Ali Mendjeli estun morceau de ville s'étendant sur une surface d'urbanisation de plus de 1200 h, hébergeant environ 180000 habitants.

Elle est très convoitée par tous les acteurs sociaux. Car en même temps qu'elle assiste à un essor fulgurant de ses activités de commerces et de services, elle reçoit d'intenses mouvements résidentiels dont les contenus sociaux se sont largement diversifiés - après un peuplement constitué presque essentiellement de bidonvillois et d'habitants pauvres de la médina qui y furent relogés par les autorités, le relais a été pris par des membres des classes moyennes auxquelles des logements en location-vente sont désormais proposés.

Cette thèse tented'identifier, puis de comprendre, le contexte et les modalités qui avaient permis de "fabriquer", puis de faire évoluer la centralité dans la Ville Nouvelle Ali Mendjeli, une nouvelle centralité dans (et à partir de) une périphérie récente d'une grande ville algérienne - la centralité étant considérée par nous, en l'occurrence, comme le lieu-synthèse, le lieu de cristallisation, de l'urbanité.Plus précisément, la thèse s'attache à déterminer les rôles respectifs de trois catégories d'acteurs publics et privés dans la production de cette centralité : les pouvoirs publics (administrateurs et hommes de l'art), les commerçants et prestataires de services et les citadins ordinaires.

Pour ce faire, nous avons choisi d'inscrire notre démarche méthodologique dans une géographie qu'on peut qualifier d'empirique", c'est-à-dire une géographie qui privilège et accorde la priorité au terrain. Ainsi, nous avons menu des investigations qui ont été conduites entre 2006 et 2012. Elles combinent relevés d'activités commerciales, passages de questionnaires, entretiens non directifs avec les acteurs publics, les commerçants et les 
habitants (récits de vie), observations flottantes et participantesde et dans l'espace de la Ville Nouvelle.

$\mathrm{Au}$ terme de cette recherche, les enquêtes que nous avons menues nous ont permis devérifier la "pluralité d'action" dans le processus de formation de la centralité à Ali Mendjeli, qui résulterait finalement de l'investissement, certes inégal, mais pluriel de l'ensemble des acteurs de la fabrique urbaine.Si l'impulsion de départ était bien le fait des responsables politiques, relayés par des urbanistes à leur service, le système d'acteurs s'est rapidement complexifié, du fait des stratégies des commerçants et des pratiques des résidents, "citadins ordinaires" :

Les commerçants et fournisseurs de services nous sont apparus comme ayant joué - et jouant toujours - un rôle essentiel dans la fixation de la centralité de la Ville Nouvelle, de même qu'ils sont largement responsables des dynamiques différentielles des différents sous-espaces (ou quartiers) qui la composent. Par les logiques de localisation qu'ils adoptent au sein d'Ali Mendjeli, et par leur grande réactivité aux changements, ils participent à la territorialisation de l'espace, puisque les lieux qu'ils occupent sont au centre des pratiques sociales, qu'ils servent de supports aux représentations, images et symboles que se forgent les habitant et que, in fine, ils contribuent très directement à la configuration des territoires (au moins à ceux de proximité).

Les citadins ordinaires, quant à eux, façonnent les paysages et donnent corps à l'urbanité d'Ali Mendjeli en usant de leurs compétences, par l'exercice de leurs pratiques et par le jeu de leurs représentations. Leurs compétences s'avèrent bien plus vastes qu'on ne pourrait ou voudrait le croire. Elles se manifestent, en amont, à travers la capacité des habitants à mobiliser des ressources de toutes sortes pour concrétiser leur projet résidentiel aux fins d'installation à Ali Mendjeli. Elles se vérifient, en aval, au travers de leur capacité à renouveler leur regard sur la ville en général, et sur la périphérie en particulier, à faire naître et cristalliser un sentiment d'appartenance à des lieux qui, au départ, étaient plutôt déniés, et, in fine, à développer une nouvelle identité urbaine qui, tantôt se fabrique par altération ou hybridation de l'ancienne identité héritée de la ville historique - ou construite en symbiose avec elle -, et tantôt coexiste avec elle. A cela s'ajoute la longue chaîne des pratiques urbaines, en particulier celles liées aux achats et aux sociabilités qui révèlent l'aptitude des habitants à se mouvoir dans la ville, à s'en approprier les espaces publics, à y inventer des lieux voués aux échanges sociaux et aux loisirs.

Aujourd'hui, l'action combinée de tous ces acteurs sociaux fait que s'inventent à Ali Mendjeli de nouvelles formes d'urbanité.

Fiche informative

\section{Discipline}

Géographie. Spécialité : Monde Arabe.

\section{Directeur}

Pierre Signoles

Université

François Rabelais, Tours, UMR 7324 CITERES (EMAM) 
Membres du Jury de thèse, soutenu le 23 mai 2013

- MmeBELABED-SAHRAOUI Badia : Professeur, Architecte-Urbaniste, Université de Constantine (Algérie)

- M. FREY Jean-Pierre : Professeur, Architecte-Sociologue, IUP Paris

- $\quad$ M. SEMMOUD Bouziane : Professeur, Géographe, Université Paris VIII

- Mme. SEMMOUD Nora : Professeur, Architecte-Géographe, Université François Rabelais

- $\quad$ M. SIGNOLES Pierre : Professeur émérite, Géographe, Université François Rabelais

Situation professionnelle actuelle

Directeur d'entreprise de maitrise d'Euvre

\section{Courriel de l'auteur}

lakehalahcene@yahoo.fr 